\title{
Four component synthesis of highly functionalized pyrano[2,3-c]pyrazoles from benzyl halides
}

\section{Mallappa Beerappa \& Kalegowda Shivashankar}

To cite this article: Mallappa Beerappa \& Kalegowda Shivashankar (2018) Four component synthesis of highly functionalized pyrano[2,3-c]pyrazoles from benzyl halides, Synthetic Communications, 48:2, 146-154, DOI: 10.1080/00397911.2017.1386788

To link to this article: https://doi.org/10.1080/00397911.2017.1386788

View supplementary material $\asymp$

Accepted author version posted online: 23

Oct 2017.

Published online: 22 Dec 2017.

Submit your article to this journal $\pi$

Џll Article views: 182

View Crossmark data \lceil

Citing articles: 3 View citing articles $\llbracket$ 


\title{
Four component synthesis of highly functionalized pyrano [2,3-c]pyrazoles from benzyl halides
}

\author{
Mallappa Beerappa and Kalegowda Shivashankar \\ Department of Chemistry, Bangalore University, Bangalore, Karnataka, India
}

\begin{abstract}
$\mathrm{N}$-methylmorpholine $\mathrm{N}$-oxide and silver oxide $\left(\mathrm{Ag}_{2} \mathrm{O}\right)$ oxidized four component reaction for the preparation of pyranopyrazole with different substituted pattern have been developed which provides rapid access to a library of compounds in good to excellent yields by using benzyl halide, malanonitrile/ethyl cyanoacetate, diethylacetylenedicarboxylate/ethyl acetoacetate and hydrazine hydrate as reactants. This transformation involves the breaking of one $\mathrm{C}-\mathrm{O}$ bond and formation of $2 \mathrm{C}-\mathrm{C}, 2 \mathrm{C}-\mathrm{N}$, and a $\mathrm{C}-\mathrm{O}$ bond leading to the formation of a five and six membered ring in one pot operation.
\end{abstract}

\section{GRAPHICAL ABSTRACT}

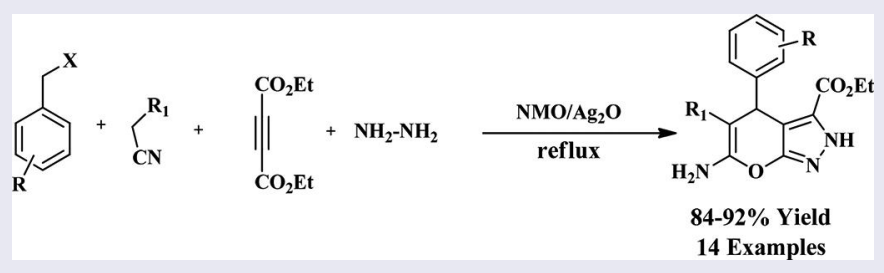

\section{ARTICLE HISTORY}

Received 25 June 2017

\section{KEYWORDS}

Multi component reactions;

$\mathrm{N}$-methylmorpholine

$\mathrm{N}$-oxide; pyran;

pyranopyrazole; pyrazole;

silver oxide

\section{Introduction}

Pollution free world is a today's main challenge for scientists. Therefore, synthetic organic chemists focused their attention toward designing reactions by following the principles of green chemistry. ${ }^{[1]}$ This has been achieved by solvent free reaction conditions that eliminates the use of hazardous solvents. ${ }^{[2]}$ Multicomponent reactions are also eco-friendly and efficient green tools as they offer significant advantage such as minimization of waste. ${ }^{[3-8]}$

Aldehydes are one of the most important substrates in variety of multicomponent reactions (MCRs). They are toxic and unstable because of aerial oxidation and polymerization. Hence, their impurities hinder the chemical reactions. ${ }^{[9]}$

Pyranopyrazole heterocyclic building blocks are of great importance in synthetic and medicinal chemistry. Both pyrazole and pyran are cyclic structural units found in many natural and synthetic products that exhibit a wide range of biological activities. ${ }^{[10]}$ It is also pertinent to note that pyranopyrazole derivatives displayed anti-inflammatory, ${ }^{[11]}$ antihypertensive, ${ }^{[12]}$ anticancer, ${ }^{[13]}$ antimicrobial, ${ }^{[14]}$ antifungal, ${ }^{[15]}$ and insecticidal 
activities. $^{[16]}$ Many commercially available drugs (Fig. 1) including celecoxib (antiinflammatory), sulfaphenazole (antibacterial), mepiprazole (antidepressant), and rimonabant (antiobesity) are derived from pyrazole core entities. ${ }^{[1]}$

Because of their useful biological activities and exceptional pharmacological properties, pyranopyrazole derivatives have attracted much attention in synthetic and medicinal chemistry. In recent years, few synthetic procedures for synthesis of pyranopyrazoles have been well documented in the literature ${ }^{[18-25]}$ among which the four component, one pot synthesis of pyranopyrazole from benzaldehyde, malanonitrile, dimethyl acetylenedicarboxylate and hydrazine hydrate is one versatile methodology. In the past years, a few methods have described the one-pot multicomponent synthesis of pyranopyrazole based on catalysts such as 1,4-diazabicyclo[2.2.2] octane (DABCO), ${ }^{[26]}$ cinchona alkaloid $^{[27]}$ and many other. ${ }^{[28-42]}$ These methods suffer from one or more disadvantages such as the use of toxic catalysts, inconsistent and poor yields and tedious workup. Hence, the development of a simple and high yielding environmentally kind procedure for the one-pot synthesis of pyranopyrazole derivatives is still reasonable.

$\mathrm{N}$-methylmorpholine $\mathrm{N}$-oxide (NMO) has received increasing attention as an inexpensive, non-toxic and readily available oxidizing agent for the synthesis of many organic compounds. ${ }^{[4-46]}$ Alkynes serve as an important synthons besides being the subunits of myriad organic compounds. Recently many reports have appeared on the utility of intra and inter molecular cyclization of alkynes in the construction of heterocycles such as (i) imidazoles, ${ }^{[47]}$ (ii) aminoindolizines, ${ }^{[48]}$ (iii) pyrrolines, ${ }^{[49]}$ (iv) iminocoumarins, ${ }^{[50]}$ (v) benzodiazepines, ${ }^{[51]}$ (vi) spirolactones, ${ }^{[52]}$ (vii) diazepines, ${ }^{[53]}$ (viii) furans, ${ }^{[54]}$ (ix) phenanthrenes, ${ }^{[55]}$ (x) pyrroles, ${ }^{[56]}$ (xi) pyridines, ${ }^{[57]}$ (xii) indolizines, ${ }^{[58]}$ and (xiii) quinolines. ${ }^{[59]}$

The pyranopyrazole skeleton has been assembled by the intermolecular cyclization of benzyl halide, malanonitrile/ethyl cyanoacetate, diethylacetylenedicarboxylate (DEAD)/ethyl acetoacetate (EAA) and hydrazine hydrate in the presence of NMO and silver oxide. To the best of our knowledge, this is the first example of synthesis of dihydropyrano[2,3-c]pyrazole derivatives directly from benzyl halides in multicomponent reaction.

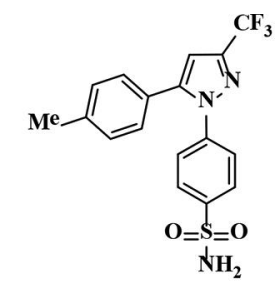

Celecoxib

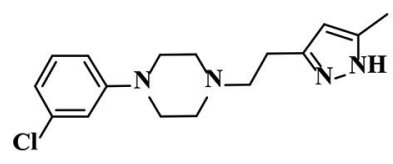

Mepiprazole

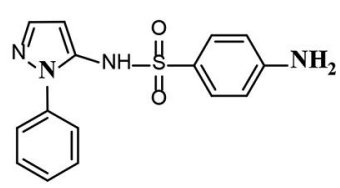

Sulfaphenazole<smiles></smiles>

Rimonabant

Figure 1. Commercially available pyrazole-derived scaffolds. 


\section{Results and discussion}

In the present work, we have initially studied the model reaction of one equivalent each of 2,6-dichlorobenzyl bromide 1a, malononitrile 2a, diethyl acetylenedicarboxylate 3a, and hydrazine hydrate 4 in the presence of NMO and silver oxide in methanol solvent under reflux condition for 90 min which afforded compound $\mathbf{5 a}$ in $70 \%$ yield. The presence of $\mathrm{Ag}_{2} \mathrm{O}$ was crucial because in its absence the reaction failed and little excess of NMO and silver oxide increases the reaction rate. Further, ethanol as solvent proved to be superior because the amine base showed better results when the MCR was performed in ethanol than any other solvents (Table 1).

The temperature effect on the product $\mathbf{5 a}$ was investigated systemically under 30, 40, 50, 60,70 , and $78{ }^{\circ} \mathrm{C}$ (reflux) in ethanol solvent. The yield reduced when the reaction proceeded toward lower temperature. Thus, reflux condition (Scheme 1) was chosen as the best temperature for the preparation of 5a. The time taken to achieve complete conversion (monitored by TLC) and the isolated yields are recorded (Table 2).

A number of structurally diverse benzyl halides were then screened for studying the generality as well as the efficacy of this present procedure. Both electron donating and electron withdrawing benzyl halides were examined and the reaction proceeded with both of them. We also found that ethyl cyanoacetate instead of malanonitrile could also be used to synthesize pyranopyrazoles successfully with excellent yields. EAA was applied in the reaction instead of diethyl acetylenedicarboxylate, which successfully led to the corresponding pyranopyrazoles in good yields. In general, the overall yield ranged from $92 \%$ for ethyl 6-amino-5-cyano-4-(4-nitrophenyl)-2,4-dihydropyrano[2,3-c]pyrazole3-carboxylate (5f) (entry 6) to $84 \%$ for 6-amino-4-(4-hydroxyphenyl)-3-methyl-2,4dihydropyrano[2,3-c]pyrazole-5-carbonitrile (5k) (entry 11).

Although we have not established the mechanism of reaction experimentally, a plausible explanation is proposed in Scheme 2 on the basis of the literature. ${ }^{[60-62]}$ In the first step,

Table 1. Solvent screening for the four component synthesis of (5a).

\begin{tabular}{clcc}
\hline Entry & Solvents & Time $(\mathrm{min})$ & Yield (\%) \\
\hline 1 & Toluene & 95 & 28 \\
2 & $\mathrm{CHCl}_{3}$ & 95 & 35 \\
3 & Dioxane & 90 & 32 \\
4 & THF & 80 & 55 \\
5 & Ethanol & 60 & 90 \\
6 & Acetonitrile & 75 & 86 \\
7 & $\mathrm{DMF}$ & 80 & 78 \\
8 & $\mathrm{H}_{2} \mathrm{O}$ & 80 & 68 \\
9 & $\mathrm{CH}_{3} \mathrm{COOH}$ & 80 & 65 \\
10 & Methanol & 90 & 70 \\
\hline
\end{tabular}

DMF, dimethylformamide; THF, tetrahydrofuran.
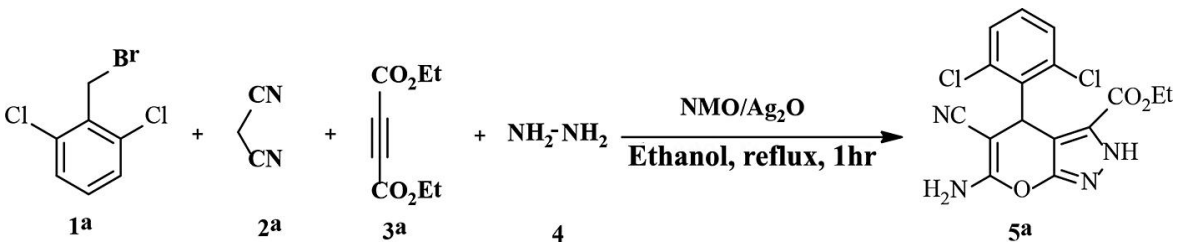

Scheme 1. Synthesis of highly functionalized pyranopyrazole (5a). 
Table 2. Scope of synthesis of pyranopyrazole derivatives (5a-n).

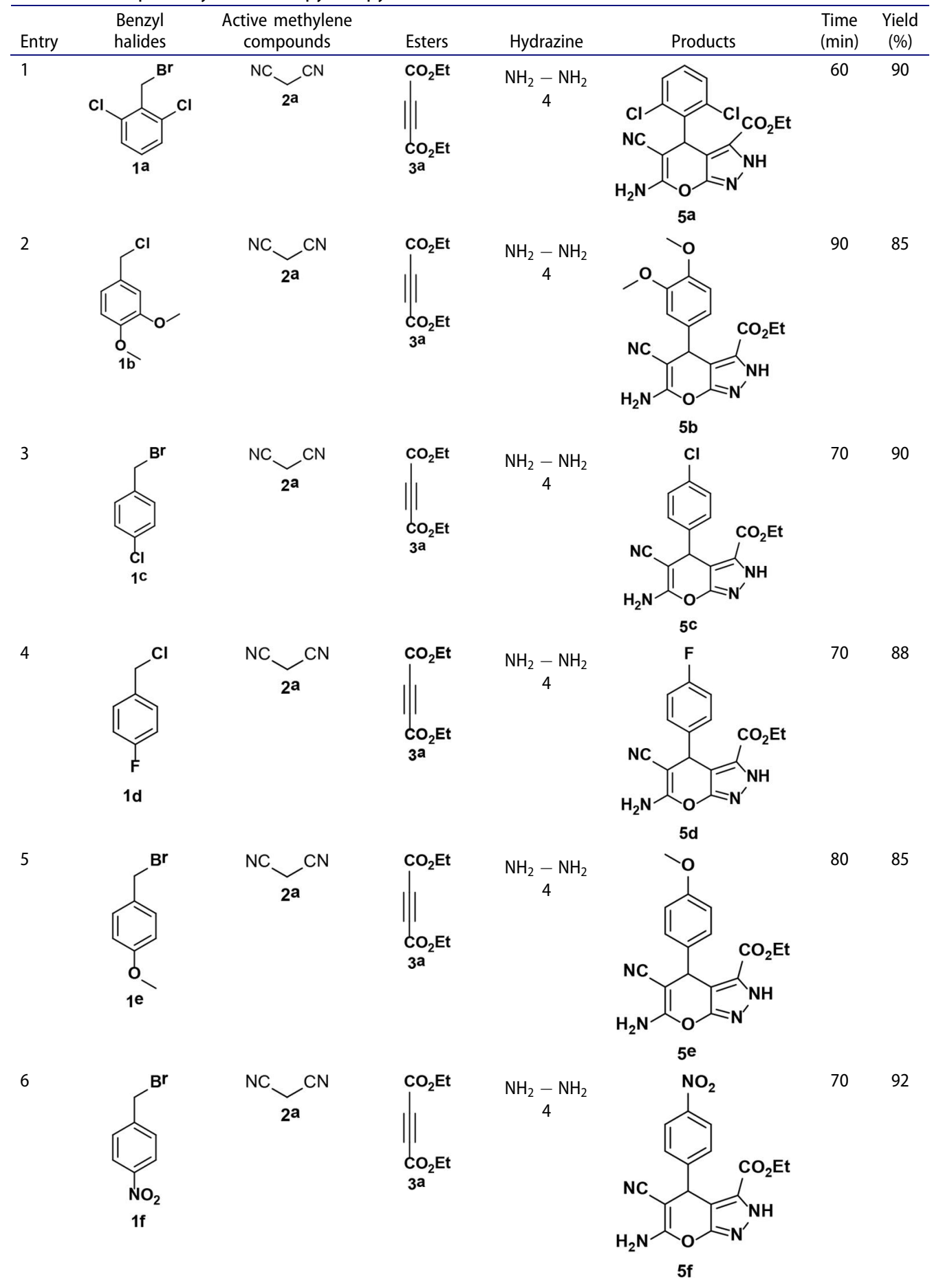


Table 2. Continued.

$\begin{array}{llllll}\text { Entry } & \begin{array}{c}\text { Benzyl } \\ \text { halides }\end{array} & \begin{array}{c}\text { Active methylene } \\ \text { compounds }\end{array} & \text { Esters } & \text { Hydrazine } & \text { Products }\end{array}$

8<smiles>Cc1ccc(CBr)c(Cl)c1</smiles><smiles>CCOC(C#CC#N)OCC</smiles><smiles>CCOC(=O)c1[nH]nc2c1C(c1ccccc1Cl)C(C#N)=C(N)O2</smiles>

$80 \quad 88$<smiles>CCOC(=O)C#CCC(=O)OCC</smiles><smiles>CCOC(=O)C1=C(N)Oc2n[nH]c(C(=O)OCC)c2C1c1c(Cl)cccc1Cl</smiles>

10<smiles>COc1cc(OC)c(CBr)c(OC)c1</smiles>

11<smiles>Oc1ccc(CBr)cc1</smiles>

$\mathrm{NC} \mathrm{CN}^{\mathrm{N}}$

$2^{a}$<smiles>CCOC(=O)CC(C)=O</smiles>

$\mathrm{NH}_{2}-\mathrm{NH}_{2}$ 4<smiles>COc1cc(OC)c(C2C(C#N)=C(N)Oc3n[nH]c(C)c32)c(OC)c1</smiles>

5 j

$\mathrm{NH}_{2}-\mathrm{NH}_{2}$<smiles>Cc1[nH]nc2c1C(c1ccc(O)cc1)C(C#N)=C(N)O2</smiles>

$\mathrm{NH}_{2}-\mathrm{NH}_{2}$<smiles>Cc1[nH]nc2c1C(c1ccccc1)C(C#N)=C(N)O2</smiles>

51
13<smiles>Clc1c[14cH][14cH]c(Cl)c1CBr</smiles>

$\mathrm{NC} \underset{\mathbf{2} \mathbf{a}}{\mathrm{CN}}$<smiles>CCCC(=O)C(C)=O</smiles>

$\mathrm{CN} \underbrace{\mathrm{CO}_{2} \mathrm{Et}}_{\mathbf{2} \mathbf{b}}$

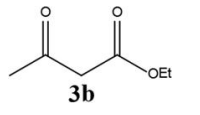

$\mathrm{NH}_{2}-\mathrm{NH}_{2}$<smiles>CCOC(=O)C1=C(N)Oc2n[nH]c(C)c2C1c1c(Cl)cccc1Cl</smiles>

$80 \quad 88$

$75 \quad 90$

$90 \quad 84$

$80 \quad 88$

$85 \quad 90$ 
Table 2. Continued.

\begin{tabular}{|c|c|c|c|c|c|c|c|}
\hline Entry & $\begin{array}{l}\text { Benzyl } \\
\text { halides }\end{array}$ & $\begin{array}{l}\text { Active methylene } \\
\text { compounds }\end{array}$ & Esters & Hydrazine & Products & $\begin{array}{l}\text { Time } \\
(\mathrm{min})\end{array}$ & $\begin{array}{l}\text { Yield } \\
(\%)\end{array}$ \\
\hline 14 & & $\mathrm{CN}_{2 \mathbf{b}}^{\mathrm{CO}_{2} \mathrm{Et}}$ & & $\begin{array}{c}\mathrm{NH}_{2}-\mathrm{NH}_{2} \\
4\end{array}$ & $\mathrm{Cl}$ & 80 & 90 \\
\hline
\end{tabular}

silver oxide assists the heterolysis of the carbon-halogen bond in the substitution reaction with NMO. The resulting silver oxide ion from the reaction between halogen and silver oxide then acts as the base in the elimination of benzaldehyde giving $N$-methylmorpholine, which in turn, in the second step would able to base catalyze Knoevenagel condensation between aldehyde and malanonitrile gives the cyanocinnamonitrile (1). The third step is the formation of pyrazolone (2) from the condensation of EAA and hydrazine. In the fourth step, Michael addition of pyrazolone (2) on cyanocinnamonitrile (1) to produce

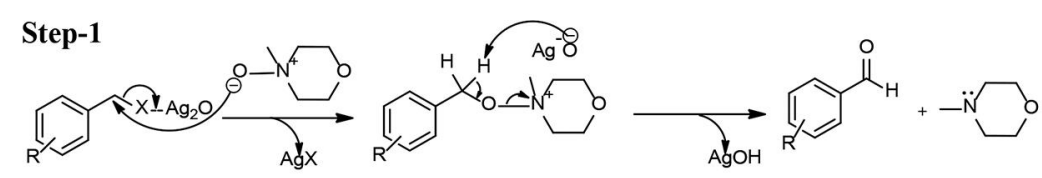

\section{Step-2}
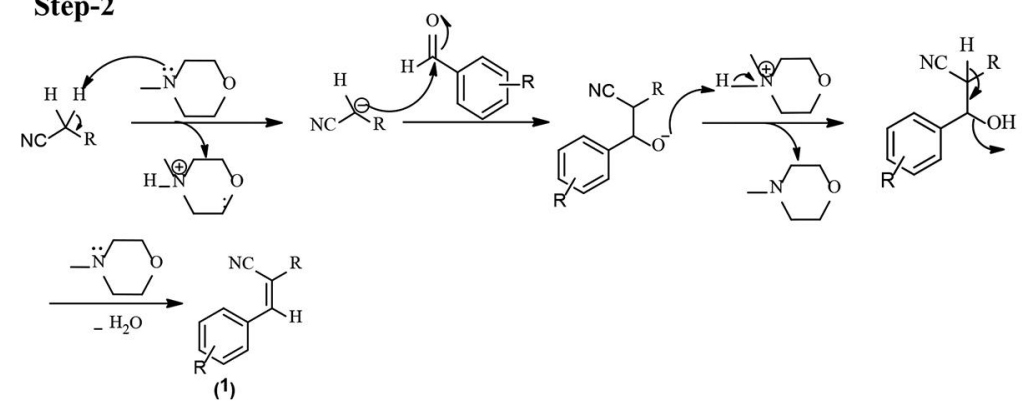

Step-3

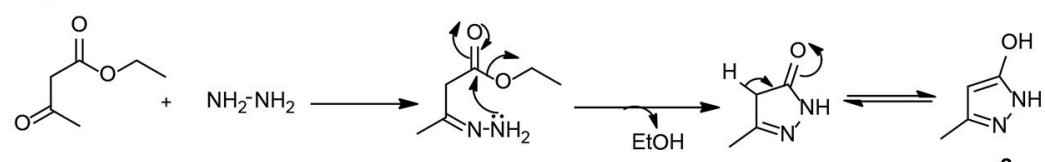

(2)

\section{Step-4}

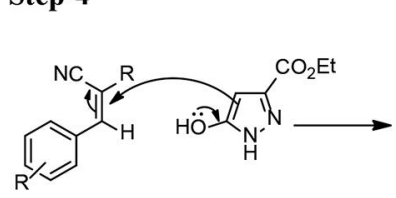

(1)

(2)

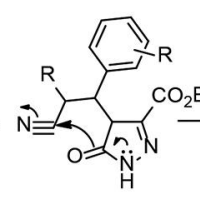

(3)

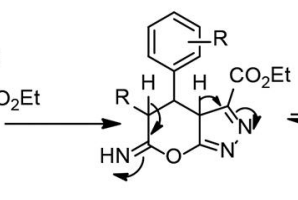

(4)<smiles></smiles>

$(5 a-n)$

Scheme 2. A plausible reaction mechanism for the formation of pyranopyrazole derivatives. 
by intermediate (3), which undergoes intra molecular cyclization by the nucleophilic addition of enolate oxygen to nitrile group to generate intermediate (4). Finally, the tautomerization of intermediate (4) gave dihydropyrano[2,3-c]pyrazoles (5a-n).

\section{Conclusion}

In short, we have developed an efficient protocol for the preparation of pyranopyrazoles through a four component reaction from benzyl halides, malanonitrile/ethyl cyanoacetate, $\mathrm{DEAD} / \mathrm{EAA}$, and hydrazine hydrate in the presence of $\mathrm{NMO}-\mathrm{Ag}_{2} \mathrm{O}$. This domino reaction implies Knoevenagel condensation, Michael addition, intramolecular cyclization and tautomerization. The mild reaction conditions, operational simplicity, broad functional group tolerance and excellent yields are the main advantages of this protocol.

\section{Experimental}

\section{General information}

Melting points were measured on an electric melting point apparatus. Elemental analysis for $\mathrm{C}, \mathrm{H}$, and $\mathrm{N}$ were performed using an Elemental Vario Micro Cube Rapid Analyzer. IR spectra were recorded on a Agilent Cary 630 FT-IR Spectrometer. ${ }^{1} \mathrm{H}$ NMR $(400 \mathrm{MHz})$ and ${ }^{13} \mathrm{C}$ NMR $(100 \mathrm{MHz})$ spectra were obtained in DMSO- $d_{6}$ as solvent and TMS as an internal standard using Bruker spectrometer. Chemical shifts were expressed in part per million (ppm). Mass spectra were recorded on a LC-MS Thermo LCQ Fleet.

\section{Typical procedure for the synthesis of ethyl 6-amino-5-cyano-4-(2,6- dichlorophenyl)-2,4-dihydropyrano[2,3-c]pyrazole-3-carboxylate (5a)}

$\mathrm{N}$-methylmorpholine $\mathrm{N}$-oxide $(110 \mathrm{mg}, 1.0 \mathrm{mmol})$ was added to a stirred solution of 2,6-dichloro benzyl bromide (200 mg, $0.84 \mathrm{mmol}$ ), malanonitrile (50 mg, $0.84 \mathrm{mmol}$ ), and silver oxide $(230 \mathrm{mg}, 1.0 \mathrm{mmol})$ in ethanol $(10 \mathrm{~mL})$ taken in a round-bottomed flask fitted with a reflux condenser and a guard tube. The resulting reaction mixture was heated at reflux condition. After half an hour, hydrazine hydrate $(40 \mathrm{mg}, 0.84 \mathrm{mmol})$ and diethyl acetylenedicarboxylate $(160 \mathrm{mg}, 0.8 \mathrm{mmol})$ were added. The mixture was stirred with a bar magnet until completion of the reaction as indicated by TLC. After cooling, the solid precipitated from the reaction mixture was filtered, washed with cold ethanol and recrystallized from ethanol.

\section{Ethyl 6-amino-5-cyano-4-(2,6-dichlorophenyl)-2,4-dihydropyrano[2,3-c]pyrazole- 3-carboxylate (5a)}

White solid; mp 231-232 ${ }^{\circ} \mathrm{C}$; IR (ATR, $\left.\mathrm{cm}^{-1}\right)$ : $3396\left(\mathrm{NH}_{2}\right), 3233(\mathrm{NH}), 2201(\mathrm{CN}), 1698$ $(\mathrm{C}=\mathrm{O}) ;{ }^{1} \mathrm{H}$ NMR $\left(400 \mathrm{MHz}, \mathrm{DMSO}-d_{6}\right): \delta=1.12\left(\mathrm{t}, J=8 \mathrm{~Hz}, 3 \mathrm{H}, \mathrm{CH}_{3}\right), 3.83(\mathrm{~m}, J=4 \mathrm{~Hz}$, $\left.2 \mathrm{H}, \mathrm{CH}_{2}\right), 4.94(\mathrm{~s}, 1 \mathrm{H}, \mathrm{C}-\mathrm{H}), 7.16-7.86\left(\mathrm{~m}, 5 \mathrm{H}, 3 \mathrm{ArH}\right.$, and $\left.\mathrm{NH}_{2}\right), 11.96(\mathrm{~s}, 1 \mathrm{H}, \mathrm{NH}) \mathrm{ppm}$; ${ }^{13} \mathrm{C}$ NMR (100 MHz, DMSO- $\left.d_{6}\right): \delta=14.0,37.0,53.3,59.0,99.5,114.5,126.3,129.5,129.7$, $130.3,135.0,135.2,142.7,153.0,158.4,161.3$ ppm; LCMS $(\mathrm{M}+\mathrm{H})=m / z 379.0$; Anal. calcd for $\mathrm{C}_{16} \mathrm{H}_{12} \mathrm{Cl}_{2} \mathrm{~N}_{4} \mathrm{O}_{3}: \mathrm{C}, 50.68 ; \mathrm{H}, 3.19 ; \mathrm{N}, 14.78$ found: C, 50.62; H, 3.11; N, $14.72 \%$. 


\section{References}

[1] Prajapati, N. P.; Vekariya, R. H.; Patel, H. D. Synth. Commun. 2015, 45, 2399-2425.

[2] Vekariya, R. H.; Patel, H. D. Synth. Commun. 2014, 45, 1031-1054.

[3] Ambethkar, S.; Padmini, V.; Bhuvanesh, N. J. Adv. Res. 2015, 6, 975-985.

[4] Beerappa, M.; Shivashankar, K. Synth. Commun. 2016, 46, 421-432.

[5] Beerappa, M.; Shivashankar, K. RSC Adv. 2015, 5, 30364-30371.

[6] Jagadishbabu, N.; Shivashankar, K. RSC Adv. 2015, 5, 95240-95246.

[7] Shamala, D.; Shivashankar, K.; Chandra Mahendra, M. Synth. Commun. 2016, 46, 433-441.

[8] Dehghan, M.; Davoodnia, A.; Bozorgmehr, M. R.; Bamoharram, F. F. Org. Prep. Proc. Int. 2017, 49, 236-248.

[9] Garima; Srivastava, V. P.; Yadav, L. D. S. Tetrahedron Lett. 2010, 51, 6436-6438.

[10] (a) Fischer, D. S.; Allan, G. M.; Bubert, C.; Vicker, N.; Smith, A.; Tutill, H. J.; Purohit, A.; Wood, L.; Packham, G.; Mahon, M. F.; J. Med. Chem. 2005, 48, 5749-5770; (b) Yang, Y. S.; Li, Q. S.; Sun, S.; Zhang, Y. B.; Wang, X. L.; Zhang, F.; Tang, J. F.; Zhu, H. L. Bioorg. Med. Chem. 2012, 20, 6048-6058.

[11] Zaki, M. E. A.; Soliman, H. A.; Hiekal, O. A.; Rashad, A. E. Z. Naturforsch. C 2006, 61, 1-5.

[12] Ahluwalia, V. K.; Dahiya, A. Indian J. Chem. 1997, 36B, 88-90.

[13] Wang, J. L.; Liu, D.; Zhang, Z. J.; Shan, S.; Han, X.; Srinivasula, S. M.; Croce, C. M.; Alnemri, E. S.; Huang, Z. Proc. Natl. Acad. Sci. USA 2000, 97, 7124-7129.

[14] El-Tamany, E. S.; El-Shahed, F. A.; Mohamed, B. H. J. Serb. Chem. Soc. 1999, 64, 9-18.

[15] Feurer, A.; Luithle, J.; Wirtz, S.; Koenig, G.; Stasch, J.; Stahl, E.; Schereiber, R.; Wunder, F.; Lang, D. Chem. Abstr. 2004, 140, 146-157.

[16] Ismail, Z. H.; Aly, G. M.; El-Degwi, M. S.; Herba, H. I.; Ghorab, M. M. J. Egypt Biol. 2003, 13, 73-76.

[17] Kamel, M. M. Acta Chim. Slov. 2015, 62, 136-147.

[18] Myrboh, B.; Mecadon, H.; Rohman, M. R.; Rajbangshi, M.; Kharkongor, I.; Laloo, B. M.; Kharbangar, I.; Kshiar, B. Org. Prep. Proc. Int. 2013, 45, 253-303.

[19] Shaabani, A.; Sarvary, A.; Rezayan, A. H.; Keshipour, S. Tetrahedron 2009, 65, 3492-3495.

[20] Enders, D.; Grossmann, A.; Gieraths, B.; Deuzdemir, M.; Merkens, C. Org. Lett. 2012, 14, 4254-4257.

[21] Zonouz, A. M.; Eskandari, I.; Khavasi, H. R. Tetrahedron Lett. 2012, 53, 5519-5522.

[22] Pore, D. M.; Patil, P. B.; Gaikwad, D. S.; Hegade, P. G.; Patil, J. D.; Undale, K. A. Tetrahedron Lett. 2013, 54, 5876-5878.

[23] Kanchithalaivan, S.; Sivakumar, S.; Kumar, R. R.; Elumalai, P.; Ahmed, Q. N.; Padala, A. K. ACS Comb. Sci. 2013, 15, 631-638.

[24] Zou, Y.; Hu, Y.; Liu, H.; Shi, D. ACS Comb. Sci. 2012, 14, 38-43.

[25] Deng, G.; Wang, F.; Lu, S.; Cheng, B. Org. Lett. 2015, 17, 4651-4653.

[26] Keyume, A.; Esmayil, Z.; Wang, L.; Jun, F. Tetrahedron 2014, 70, 3976-3980.

[27] Gogoi, S.; Zhao, C. G. Tetrahedron Lett. 2009, 50, 2252-2255.

[28] Tayade, Y. A.; Padvi, S. A.; Wagh, Y. B.; Dalal, D. S. Tetrahedron Lett. 2015, 56, 2441-2447.

[29] Moeinpour, F.; Khojastehnezhad, A. Chin. Chem. Lett. 2015, 26, 575-579.

[30] Shinde, P. V.; Gujar, J. B.; Shingate, B. B.; Shingare, M. S. Bull. Korean Chem. Soc. 2012, 33, 41-45.

[31] Bihani, M.; Bora, P. P.; Bez, G.; Askari, H. ACS Sustainable Chem. Eng. 2013, 1, 440-447.

[32] Litvinov, Y. M.; Shestopalov, A. A.; Rodinovskaya, L. A.; Shestopalov, A. M. J. Comb. Chem. 2009, 11, 914-919.

[33] Saha, A.; Payra, S.; Banerjee, S. Green Chem. 2015, 17, 2859-2866.

[34] Kiyania, H.; Samimib, H. A.; Ghorbania, F.; Esmaieli, S. Curr. Chem. Lett. 2013, 2, 197-206.

[35] Tamaddona, F.; Farahia, M.; Karamib, B. J. Mol. Catal. A: Chem. 2012, 356, 85-89.

[36] Babaie, M.; Sheiban, H. Arab. J. Chem. 2011, 4, 159-162.

[37] Wu, M.; Feng, Q.; Wan, D.; Ma, J. Synth. Commun. 2013, 43, 1721-1726.

[38] Aleem, M. A. E.; El-Remaily, A. A. Tetrahedron 2014, 70, 2971-2975.

[39] Guo, R. Y.; An, Z. M.; Mo, L. P.; Yang, S. T.; Liu, H. X.; Wang, S. X.; Zhang, Z. H. Tetrahedron 2013, 69, 9931-9938. 
[40] Muramulla, S.; Zhao, C. G. Tetrahedron Lett. 2011, 52, 3905-3908.

[41] Muthusaravanan, S.; Perumal, S.; Yogeeswari, P.; Sriram, D. Tetrahedron Lett. 2010, 51, 6439-6443.

[42] Khurana, J. M.; Nand, B.; Kumar, S. Synth. Commun. 2011, 41, 405-410.

[43] Schmidt, A. K. C.; Stark, C. B. W. Org. Lett. 2011, 13, 4164-4167.

[44] Ley, S. V.; Ramarao, C.; Lee, A. L.; Ostergaard, N.; Smith, S. C.; Shirley, I. M. Org. Lett. 2003, 5, 187-187.

[45] Choudary, B. M.; Chowdari, N. S.; Jyothi, K.; Kantam, M. L. J. Am. Chem. Soc. 2002, 124, 5341-5349.

[46] Nicolaou, K. C.; Adsool, V. A.; Hale, C. R. H. Org. Lett. 2010, 12, 1552-1555.

[47] Guchhait, S. K.; Chandgude, A. L.; Priyadarshani, G. J. Org. Chem. 2012, 77, 4438-4444.

[48] Yan, B.; Liu, Y. Org. Lett. 2007, 9, 4323-4326.

[49] Cui, S. L.; Wang, J.; Wang, Y. G. Org. Lett. 2007, 9, 5023-5025.

[50] Cui, S. L.; Lin, X. F.; Wang, Y. G. Org. Lett. 2006, 8, 4517-4520.

[51] Maiti, G.; Kayal, U.; Karmakar, R.; Bhattacharya, R. N. Tetrahedron Lett. 2012, 53, 1460-1463.

[52] Kiruthika, S. E.; Amritha, R.; Perumal, P. T. Tetrahedron Lett. 2012, 53, 3268-3273.

[53] Maleki, A. Tetrahedron Lett. 2013, 54, 2055-2059.

[54] Silwal, S.; Rahaim, R. J. Tetrahedron Lett. 2015, 56, 5738-5742.

[55] Saifuddin, M.; Agarwal, P. K.; Kundu, B. J. Org. Chem. 2011, 76, 10122-10128.

[56] Estevez, V.; Villacampa, M.; Menendez, J. C. Chem. Soc. Rev. 2010, 39, 4402-4421.

[57] Sha, F.; Huang, X. Angew. Chem. 2009, 121, 3510-3513.

[58] Sandeep, C.; Padmashali, B.; Kulkarni, R. S. Tetrahedron Lett. 2013, 54, 6411-6414.

[59] Bala, B. D.; Muthusaravanan, S.; Perumal, S. Tetrahedron Lett. 2013, 54, 3735-3739.

[60] Khumraksa, B.; Phakhodee, W.; Pattarawarapan, M. Tetrahedron Lett. 2013, 54, 1983-1986.

[61] Pal, S.; Khan, M. N.; Karamthulla, S.; Abbas, S. J.; Choudhury, L. H. Tetrahedron 2013, 54, 5434-5440.

[62] Kanagaraj, K.; Pitchumani, K. Tetrahedron Lett. 2010, 51, 3312-3316. 